\title{
SINGULARITIES OF LAGRANGIAN MEAN CURVATURE FLOW: MONOTONE CASE
}

\begin{abstract}
ANDRÉ NeVES
ABSTRACT. We study the formation of singularities for the mean curvature flow of monotone Lagrangians in $\mathbb{C}^{n}$. More precisely, we show that if singularities happen before a critical time then the tangent flow can be decomposed into a finite union of areaminimizing Lagrangian cones (Slag cones). When $n=2$, we can improve this result by showing that each connected components of the rescaled flow converge to an areaminimizing cone, as opposed to possible non-area minimizing union of Slag cones. In the last section, we give specific examples for which such singularity formation occurs.
\end{abstract}

\section{Introduction}

We study the formation of singularities for Lagrangian mean curvature flow when the initial condition is a Lagrangian in $\mathbb{C}^{n}$ for which the Liouville-class equals the Maslov-class. The standard examples of such Lagrangians are the Clifford torus in $\mathbb{C}^{2}$ which, under mean curvature flow, shrink to a point at time $1 / 2$. In this paper, we show that if the singularity occurs for some time $T<1 / 2$, then the tangent flow at the singularity has the same properties as the tangent flow at a singularity when the initial condition is a zero Maslov-class Lagrangian (see [4]). More precisely, we show that the tangent flow decomposes into a finite union of Slag cones (Theorem A) and, when $n=2$, we improve this result and show that connected components of the rescaled flow converge to a single SLag cone (Theorem B) as opposed to a union of SLag cones which might have different Lagrangian angles and hence not be area-minimizing. If this property did not hold, it would be very difficult to obtain any kind of regularity theory for the Lagrangian at the singular time $T$. For instance, the optimal regularity result expected for the flow of Lagrangian surfaces is that singularities are isolated. Furthermore, the size of the singular set of blow-ups is never smaller than the size of the singular set of the surface at the singular time. These two facts together suggest that if one wants to develop a regularity theory for Lagrangian mean curvature flow, then we should show first that the singular set of blow-ups consists at most of isolated points. Theorem B says that is indeed the case because the set where SLag cones are not smooth are isolated.

A similar result was obtained in [4] in the context of rational Lagrangians having oscillation of the Lagrangian angle smaller than $\pi$.

In the last section, we give examples of Lagrangians which are Hamiltonian isotopic to a Clifford torus and for which the type of singularities described above occurs (Theorem C). Similar examples were also constructed in [1] where it is shown that Type I singularities do not occur for the flow of monotone Lagrangians before a certain

Received by the editors March 12, 2008.

The author was partially supported by NSF grant DMS-06-04164. 
predetermined time. In addition, in [1] the authors also study the behavior of the flow assuming some rotational symmetry for the initial condition.

The organization of the paper is as follows. In the next section we introduce notation and derive some evolution equations. In Section 3 we state Theorem A, Theorem B, and Theorem C. Section 4 is devoted to the proof of Theorem A and in Section 5 we prove Theorem B. Theorem $\mathrm{C}$ is proven in Section 6.

Acknowledgments The author would like to thank $\mathrm{Mu}$-Tao Wang for many interesting discussions regarding the use of the Liouville form in the field. The author is also thankful to the referee for many useful suggestions.

\section{Preliminaires}

The standard complex structure and standard symplectic form on $\mathbb{C}^{n}$ are denoted by $J$ and $\omega$ respectively. We consider also the closed complex-valued $n$-form given by

$$
\Omega \equiv d z_{1} \wedge \ldots \wedge d z_{n}
$$

and the Liouville form given by

$$
\lambda \equiv \sum_{i=1}^{n} x_{i} d y_{i}-y_{i} d x_{i}, \quad d \lambda=2 \omega
$$

where $z_{j}=x_{j}+i y_{j}$ are complex coordinates for $\mathbb{C}^{n}$.

A smooth $n$-dimensional submanifold $L$ in $\mathbb{C}^{n}$ is called Lagrangian if $\omega_{L}=0$ and this implies that (see, for instance, [2])

$$
\Omega_{L}=\exp (i \theta) \operatorname{vol}_{L},
$$

where $\operatorname{vol}_{L}$ denotes the volume form of $L$ and the multivalued function $\theta$ is called the Lagrangian angle. Nevertheless, $d \theta$ is a well defined closed form on $L$ and its cohomology class is denoted by Maslov-class. If $\theta \equiv \theta_{0}$, then $L$ is calibrated by

$$
\operatorname{Re}\left(\exp \left(-i \theta_{0}\right) \Omega\right)
$$

and hence area-minimizing. In this case, $L$ is referred as being Special Lagrangian.

Note that the Lagrangian condition implies that $\lambda$ is a closed form on $L$. A Lagrangian $L$ is said to be monotone if

$$
[\lambda]=c[d \theta]
$$

for some positive constant $c$. When $c=1$ we call $L$ a normalized monotone Lagrangian and note that every monotone Lagrangian can be rescaled to a normalized one. The standard examples of monotone Lagrangians in $\mathbb{C}^{2}$ are the Clifford torus

$$
T_{r} \equiv\left\{\left(z_{1}, z_{2}\right) \in \mathbb{C}|| z_{1} \mid=r \text { and }\left|z_{2}\right|=r\right\} .
$$

The Clifford torus is normalized monotone when $r=1$.

Let $L_{0}$ be a monotone Lagrangian and denote by $\left(L_{t}\right)_{0 \leq t<T}$ a solution to Lagrangian mean curvature flow. Denoting by $F_{t}$ the normal deformation by mean 
curvature, we have

$$
\begin{aligned}
\left.\frac{d}{d t} \int_{F_{t}(\gamma)} \lambda=\frac{d}{d t} \int_{\gamma} F_{t}^{*} \lambda=\int_{\gamma} \mathcal{L}_{H} F_{t}^{*} \lambda=\int_{\gamma} d F_{t}^{*}(H\lrcorner \lambda\right) & \left.F_{t}^{*}(H\lrcorner 2 \omega\right) \\
& =-2 \int_{\gamma} d F_{t}^{*} \theta_{t}=-2\left[d \theta_{t}\right]
\end{aligned}
$$

for every $[\gamma]$ in $H_{1}\left(L_{t}\right)$. Therefore, the monotone condition is preserved under the flow and we can combine this with the fact that $\left[d \theta_{t}\right]$ is in $H^{1}\left(L_{t}, 2 \pi \mathbb{Z}\right)$ in order to obtain

Lemma 2.1. For all $t<T$,

$$
[\lambda]=(c-2 t)\left[d \theta_{t}\right] \quad \text { in } H^{1}\left(L_{t}\right) .
$$

This computation was communicated to the author by Smoczyk and can be seen as a higher codimension analogue of the fact that curve shortening flow decreases enclosed area linearly. We obtain from Lemma 2.1 the existence of smooth functions $\gamma_{t}$ on $L_{t}$ such that

$$
d \gamma_{t}=\lambda+(2 t-c) d \theta_{t}
$$

or, equivalently,

$$
\nabla \gamma_{t}=(J x)^{\top}+\nabla \theta_{t}
$$

where $(J x)^{\top}$ denotes the tangential projection on $T_{x} L_{t}$. The following computation can also be found in [1].

Proposition 2.2. The functions $\gamma_{t}$ can be chosen so that

$$
\frac{d \gamma_{t}}{d t}=\Delta \gamma_{t}
$$

Proof. Assume without loss of generality that the family of functions $\left(\gamma_{t}\right)$ is smooth with respect to the time parameter. Denoting by $d^{*}$ the negative adjoint of $d$, it is know that $d \theta_{t}$ satisfies the equation (see [6])

$$
\frac{d\left(d \theta_{t}\right)}{d t}=d d^{*}\left(d \theta_{t}\right)
$$

Furthermore, we can see from [4, Lemma 6.2] that

$$
\left.d^{*} \lambda=H\right\lrcorner \lambda
$$

from which it follows that

$$
\left.\left.\frac{d \lambda}{d t}=\mathcal{L}_{H} \lambda=d(H\lrcorner \lambda\right)+H\right\lrcorner 2 \omega=d d^{*} \lambda-2 d \theta_{t} .
$$

Combining this with identity (1), we obtain that

$$
\frac{d\left(d \gamma_{t}\right)}{d t}=d d^{*}\left(\lambda+(2 s-c) d \theta_{t}\right)=d \Delta \gamma_{t}
$$

and thus, we can add a time dependent constant to each $\gamma_{t}$ so that the desired result follows. 


\section{Statement of results}

If we consider a solution to mean curvature flow $\left(L_{t}\right)_{0 \leq t<T}$ for which the initial condition is a normalized monotone Lagrangian, then Lemma 2.1 shows that some sort of singular behavior happens at time $t=1 / 2$. We focus in this paper on singularities that happen at the point $\left(x_{0}, T\right)$ in space-time with $T<1 / 2$. The results we present next show that these type of singularities have the same behavior as finite time singularities for the mean curvature flow of zero Maslov-class Lagrangians. We now give an heuristic argument for why this phenomena is somehow expected.

Consider any point $x_{1}$ in $\mathbb{C}^{n}$ and define

$$
\bar{L}_{s} \equiv \exp (s)\left(L_{(1-\exp (-2 s)) / 2}-x_{1}\right) \text {. }
$$

Then, $\bar{L}_{s}$ solves

$$
\frac{d x}{d s}=H+\mathbf{x}^{\perp},
$$

where $\mathbf{x}^{\perp}$ denotes the orthogonal projection on $\left(T_{x} \bar{L}_{s}\right)^{\perp}$ of the vector determined by the point $x$ in $\mathbb{C}^{n}$. The change of basepoint does not change the cohomology class of $\lambda$ and so, we obtain by Lemma 2.1 that $\bar{L}_{s}$ is a normalized monotone Lagrangian. This implies the existence of smooth functions $\bar{\gamma}_{s}$ such that, using Proposition 2.2,

$$
J \nabla \bar{\gamma}_{s}=H+\mathbf{x}^{\perp} \quad \text { and } \quad \frac{d \bar{\gamma}_{s}}{d s}=\Delta \bar{\gamma}_{s}+2 \gamma_{s} .
$$

This computations highlights the analogy between the family $\left(\bar{L}_{s}\right)$ and a solution to mean curvature flow of zero Maslov-class Lagrangians. Note that $\left(\bar{L}_{s}\right)$ exist for all time precisely when $T=1 / 2$ and so, arguing heuristically, finite-time singularities for zero Maslov-class Lagrangians "correspond" to singularities at time $T<1 / 2$ for normalized monotone Lagrangians.

In order to study finite time singularities for mean curvature flow, take any sequence $\left(\sigma_{j}\right)$ going to infinity and consider

$$
L_{s}^{j} \equiv \sigma_{j}\left(L_{T+s / \sigma_{j}^{2}}-x_{0}\right) \quad \text { for }-\sigma_{j}^{2} T<s<0,
$$

which is still a solution to Lagrangian mean curvature flow (called the rescaled flow). For the rest of this paper, the initial condition for the flow $L_{0}$ will have the property that, for some constant $C_{0}$,

$$
\mathcal{H}^{n}\left(L_{0} \cap B_{R}(0)\right) \leq C_{0} R^{n}
$$

for all $R>0$

Arguing informally, the following theorem states that a sequence of rescaled flows at a singularity converges weakly to a finite union of integral Special Lagrangian cones.

Theorem A. Let $L_{0}$ be a normalized monotone Lagrangian in $\mathbb{C}^{n}$ for which the Lagrangian mean curvature flow develops a singularity at time $T<1 / 2$. For any sequence of rescaled flows $\left(L_{s}^{j}\right)_{s<0}$ at a singularity, there exists a finite set of Lagrangian angle

$$
\left\{\exp \left(i \bar{\theta}_{1}\right), \ldots, \exp \left(i \bar{\theta}_{N}\right)\right\}
$$

and integral Special Lagrangian cones

$$
L_{1}, \ldots, L_{N}
$$


such that, after passing to a subsequence, we have for every smooth function $\phi$ with compact support, every $f$ in $C^{2}\left(S^{1}\right)$, and every $s<0$,

$$
\lim _{j \rightarrow \infty} \int_{L_{s}^{j}} f\left(\exp \left(i \theta_{j, s}\right)\right) \phi d \mathcal{H}^{n}=\sum_{k=1}^{N} m_{k} f\left(\exp \left(i \bar{\theta}_{k}\right)\right) \mu_{k}(\phi)
$$

where $\mu_{k}$ and $m_{k}$ denote the Radon measure of the support of $L_{k}$ and its multiplicity respectively.

Furthermore, the set $\left\{\exp \left(i \bar{\theta}_{1}\right), \ldots, \exp \left(i \bar{\theta}_{N}\right)\right\}$ does not depend on the sequence of rescalings chosen.

When $n=2$ we can strengthen this result and show that on each connected components of $L_{s}^{j} \cap B_{R}(0)$ the Lagrangian angle converges to a constant. Arguing heuristically, this property shows that the formation of singularities exhibits a "nice" behavior. A similar property was proven in [4] in the context of rational Lagrangians in $\mathbb{C}^{n}$ with oscillation of the Lagrangian angle less than $\pi$.

Theorem B. Let $L_{0}$ be a normalized monotone Lagrangian in $\mathbb{C}^{2}$ for which the Lagrangian mean curvature flow develops a singularity at time $T<1 / 2$. The following property holds for all $R>0$ and almost all $s<0$.

For any sequence $\Sigma_{j}$ of connected components of $B_{4 R}(0) \cap L_{s}^{j}$ that intersect $B_{R}(0)$, there exists a Special Lagrangian cone $\Sigma$ in $B_{2 R}(0)$ with Lagrangian angle $\bar{\theta}$ such that, after passing to a subsequence,

$$
\lim _{j \rightarrow \infty} \int_{\Sigma_{j}} f\left(\exp \left(i \theta_{j, s}\right)\right) \phi d \mathcal{H}^{n}=m f(\exp (i \bar{\theta})) \mu(\phi)
$$

for every $f$ in $C^{2}$ rem $\left(S^{1}\right)$ and every smooth $\phi$ compactly supported in $B_{2 R}(0)$, where $\mu$ and $m$ denote the Radon measure of the support of $L$ and its multiplicity respectively.

Without the connectedness assumption on each $\Sigma_{j}$ the theorem would fail for trivial reasons. The requirement that $\Sigma_{j}$ intersects $B_{R}(0)$ is necessary in order to prevent its area to converge to zero.

From the proof of Theorem $A$, it follows that for almost all $s<0$ and all $R>0$,

$$
\lim _{j \rightarrow \infty} \int_{L_{s}^{j} \cap B_{R}(0)}\left|\nabla \theta_{j, s}\right|^{2} d \mathcal{H}^{2}=0 .
$$

We remark that this alone is not enough to show Theorem B because the sequence of smooth manifolds $L_{s}^{j}$ are becoming singular when $j$ goes to infinity and so, no Poincaré inequality holds with a constant independent of $j$. This means we cannot conclude that, on each connected component of $L_{s}^{i}$, the Lagrangian angles $\theta_{i, s}$ converge to a constant. To overcome this obstacle we will need to explore the fact that a function $\gamma_{t}$ satisfying (1) is well defined.

Finally, assuming some rotational symmetry, we construct examples that show that the type of singularities considered in Theorem A and Theorem B occur.

Theorem C. There is a normalized monotone Lagrangian $L_{0}$ which is Hamiltonian isotopic to a Clifford torus and for which the Lagrangian mean curvature flow develops a singularity at the origin at time $T<1 / 2$. The rescaled flow is a transverse union of two Lagrangian planes with the same Lagrangian angle. 


\section{Proof of Compactness Theorem A}

Before proving Theorem A we recall Huisken's monotonicity formula [3] valid for any smooth family of $k$-dimensional submanifolds $\left(N_{t}\right)_{t \geq 0}$ moving by mean curvature flow in $\mathbb{R}^{m}$. Consider the backward heat kernel

$$
\Phi_{x_{0}, T}(x, t)=\frac{1}{(4 \pi(T-t))^{k / 2}} e^{-\frac{\left|x-x_{0}\right|^{2}}{4(T-t)}} .
$$

When $\left(x_{0}, T\right)=(0,0)$, we denote it simply by $\Phi$. The following formula holds

$$
\frac{d}{d t} \int_{N_{t}} f_{t} \Phi_{x_{0}, T}=\int_{N_{t}}\left(\frac{d}{d t} f_{t}-\Delta f_{t}-\left|H+\frac{\left(\mathbf{x}-\mathbf{x}_{0}\right)^{\perp}}{2(T-t)}\right|^{2} f_{t}\right) \Phi_{x_{0}, T}
$$

where $f_{t}$ is a smooth function with polynomial growth at infinity and $\left(\mathbf{x}-\mathbf{x}_{0}\right)^{\perp}$ denotes the orthogonal projection on $\left(T_{x} N\right)^{\perp}$ of the vector determined by the point $\left(x-x_{0}\right)$ in $\mathbb{R}^{m}$.

The aim of this section is to prove Theorem A, which we restate it for the sake of convenience.

Theorem A. Let $L_{0}$ be a normalized monotone Lagrangian in $\mathbb{C}^{n}$ for which the Lagrangian mean curvature flow develops a singularity at time $T<1 / 2$. For any sequence of rescaled flows $\left(L_{s}^{j}\right)_{s<0}$ at a singularity, there exists a finite set of Lagrangian angle

$$
\left\{\exp \left(i \bar{\theta}_{1}\right), \ldots, \exp \left(i \bar{\theta}_{N}\right)\right\}
$$

and integral Special Lagrangian cones

$$
L_{1}, \ldots, L_{N}
$$

such that, after passing to a subsequence, we have for every smooth function $\phi$ with compact support, every $f$ in $C^{2}\left(S^{1}\right)$, and every $s<0$

$$
\lim _{j \rightarrow \infty} \int_{L_{s}^{j}} f\left(\exp \left(i \theta_{j, s}\right)\right) \phi d \mathcal{H}^{n}=\sum_{k=1}^{N} m_{k} f\left(\exp \left(i \bar{\theta}_{k}\right)\right) \mu_{k}(\phi),
$$

where $\mu_{k}$ and $m_{k}$ denote the Radon measure of the support of $L_{k}$ and its multiplicity respectively.

Furthermore, the set $\left\{\exp \left(i \bar{\theta}_{1}\right), \ldots, \exp \left(i \bar{\theta}_{N}\right)\right\}$ does not depend on the sequence of rescalings chosen.

Proof. Set $u(x) \equiv\left\langle J x_{0}, x\right\rangle$, where $x_{0}$ is the point at which the singularity occurs. According to Proposition 2.2, we can choose a family of functions $\gamma_{t}$ so that

$$
d \gamma_{t}=\lambda+(2 t-1) d \theta_{t}-d u \quad \text { and } \quad \frac{d \gamma_{t}}{d t}=\Delta \gamma_{t} .
$$

A simple computation shows that

$$
\begin{aligned}
\frac{d}{d t} \int_{L_{t}} \gamma_{t}^{2} \Phi_{x_{0}, T} d \mathcal{H}^{n} & \\
& =-\int_{L_{t}}\left(2\left|\nabla \gamma_{t}\right|^{2}+\left|H-\left(\mathbf{x}-\mathbf{x}_{0}\right)^{\perp} / 2(T-t)\right|^{2} \gamma_{t}^{2}\right) \Phi_{x_{0}, T} d \mathcal{H}^{n}
\end{aligned}
$$


and hence

$$
\lim _{t \rightarrow T} \int_{t}^{T} \int_{L_{s}}\left|\nabla \gamma_{s}\right|^{2} \Phi_{x_{0}, T} d \mathcal{H}^{n} d s=0 .
$$

From Huisken's monotonicity formula we know that

$$
\lim _{t \rightarrow T} \int_{t}^{T} \int_{L_{s}}\left|H+\left(\mathbf{x}-\mathbf{x}_{0}\right)^{\perp} /(2(T-t))\right|^{2} \Phi_{x_{0}, T} d \mathcal{H}^{n} d s=0
$$

and thus, because the identity

$$
J \nabla \gamma_{t}=-\left(\mathbf{x}-\mathbf{x}_{0}\right)^{\perp}+(2 t-1) H
$$

implies that

$$
\left.|(2 T-1) H|^{2} \leq 2\left|\nabla \gamma_{t}\right|^{2}+8(T-t)^{2}\right)\left|H+\left(\mathbf{x}-\mathbf{x}_{0}\right)^{\perp} /(2(T-t))\right|^{2},
$$

we obtain

$$
\lim _{t \rightarrow T} \int_{t}^{T} \int_{L_{s}}\left(|H|^{2}+\left|\left(\mathbf{x}-\mathbf{x}_{0}\right)^{\perp} /(T-t)\right|^{2}\right) \Phi_{x_{0}, T} d \mathcal{H}^{n} d s=0 .
$$

Fix any $a<b<0$. In terms of the sequence of flows $\left(L_{s}^{j}\right)$, it follows from the above identity that

$$
\lim _{j \rightarrow \infty} 2 \int_{a}^{b} \int_{L_{s}^{j}}\left(\left|\mathbf{x}^{\perp}\right|^{2}+|H|^{2}\right) \Phi d \mathcal{H}^{n} d s=0
$$

and hence, we can choose $a<0$ so that

$$
\lim _{i \rightarrow \infty} \int_{L_{a}^{i} \cap B_{R}(0)}\left(\left|\mathbf{x}^{\perp}\right|^{2}+|H|^{2}\right) d \mathcal{H}^{n}=0
$$

for all positive $R$.

The rest of the proof follows from adapting the arguments used in the proof of [4, Theorem A]. The first step consists in showing

Proposition 4.1. Let $\left(L^{j}\right)$ be a sequence of smooth Lagrangians in $\mathbb{C}^{n}$ such that, for some fixed $R>0$, the following properties hold:

(a) There exists a constant $D_{0}$ for which

$$
\mathcal{H}^{n}\left(L^{j} \cap B_{2 R}(0)\right) \leq D_{0} R^{n}
$$

for all $j \in \mathbb{N}$;

(b)

and

$$
\lim _{j \rightarrow \infty} \mathcal{H}^{n-1}\left(\partial L^{j} \cap B_{2 R}(0)\right)=0
$$

$$
\lim _{j \rightarrow \infty} \int_{L^{j} \cap B_{2 R}(0)}|H|^{2} d \mathcal{H}^{n}=0 .
$$

Then, there exist a finite set

$$
\left\{\exp \left(i \bar{\theta}_{1}\right), \ldots, \exp \left(i \bar{\theta}_{N}\right)\right\}
$$

and integral Special Lagrangians

$$
L_{1}, \ldots, L_{N}
$$


such that, after passing to a subsequence, we have for every smooth function $\phi$ compactly supported in $B_{R}(0)$ and every $f$ in $C\left(S^{1}\right)$

$$
\lim _{j \rightarrow \infty} \int_{L^{j}} f\left(\exp \left(i \theta_{j}\right)\right) \phi d \mathcal{H}^{n}=\sum_{j=k}^{N} m_{k} f\left(\exp \left(i \bar{\theta}_{k}\right)\right) \mu_{k}(\phi),
$$

where $\mu_{k}$ and $m_{k}$ denote, respectively, the Radon measure of the support of $L_{k}$ and its multiplicity.

Proof. A slightly different version of this proposition was proven in [4, Proposition 5.1] where $L^{j}$ was assumed to be zero Maslov-class. The same proof applies straightforwardly provided we consider the sets

$$
\left\{x \in L^{j}|| \exp \left(i \theta_{j}(x)\right)-\exp \left(i \bar{\theta}_{1}\right) \mid \leq \varepsilon\right\}
$$

and the functions $\cos \theta_{j}$ instead of the sets

$$
\left\{x \in L^{j}|| \theta_{j}(x)-\bar{\theta}_{1} \mid \leq \varepsilon\right\}
$$

and the functions $\theta_{j}$ respectively.

A standard fact (see, for instance, [4, Lemma B.1]) implies the existence of a constant $D_{0}$ for which

$$
\mathcal{H}^{n}\left(L_{a}^{j} \cap B_{R}(0)\right) \leq D_{0} R^{n}
$$

for all positive $R$. Hence, we can apply Proposition 4.1 to the sequence $\left(L_{a}^{j}\right)$ and, after a simple diagonalization argument, obtain a subsequence (still indexed by $j$ ) for which there are integral Special Lagrangians

$$
L_{1}, \ldots, L_{N}
$$

and a finite set

$$
\left\{\exp \left(i \bar{\theta}_{1}\right), \ldots, \exp \left(i \bar{\theta}_{N}\right)\right\}
$$

such that for every smooth function $\phi$ compactly supported and every $f$ in $C^{2}\left(S^{1}\right)$

$$
\lim _{j \rightarrow \infty} \int_{L_{a}^{j}} f\left(\exp \left(i \theta_{j, a}\right)\right) \phi d \mathcal{H}^{n}=\sum_{j=k}^{N} m_{k} f\left(\exp \left(i \bar{\theta}_{k}\right)\right) \mu_{k}(\phi),
$$

where $\mu_{k}$ and $m_{k}$ denote, respectively, the Radon measure and the multiplicity of $L_{k}$. The fact that

$$
\lim _{k \rightarrow \infty} \int_{L_{a}^{j} \cap B_{R}(0)}\left|\mathbf{x}^{\perp}\right|^{2} d \mathcal{H}^{n}=0
$$

for all positive $R$ implies that the Special Lagrangians $L_{k}$ are all cones.

It is well known that (see, for instance, [6])

$$
\frac{d \exp \left(i \theta_{j, s}\right)}{d s}=\Delta \exp \left(i \theta_{j, s}\right)+\exp \left(i \theta_{j, s}\right)|H|^{2},
$$

and so, a simple computation shows that

$$
P_{j, s} \equiv \frac{d f\left(\exp \left(i \theta_{j, s}\right)\right)}{d s}-\Delta f\left(\exp \left(i \theta_{j, s}\right)\right)
$$

satisfies

$$
\left|P_{j, s}\right|+\left|\nabla f\left(\exp \left(i \theta_{j, s}\right)\right)\right|^{2} \leq C|H|^{2},
$$


where $C=C(f)$. Hence,

$$
\begin{aligned}
& \frac{d}{d s} \int_{L_{s}^{j}} f\left(\exp \left(i \theta_{j, s}\right)\right) \phi d \mathcal{H}^{n}=-\int_{L_{s}^{j}}\left\langle\nabla f\left(\exp \left(i \theta_{j, s}\right)\right), \nabla \phi\right\rangle d \mathcal{H}^{n} \\
&+\int_{L_{s}^{j}} f\left(\exp \left(i \theta_{j, s}\right)\right)\langle H, D \phi\rangle d \mathcal{H}^{n}+\int_{L_{s}^{j}} P_{j, s} \phi d \mathcal{H}^{n} \\
&-\int_{L_{s}^{j}} f\left(\exp \left(i \theta_{j, s}\right)\right)|H|^{2} \phi d \mathcal{H}^{n} .
\end{aligned}
$$

It follows from (4) and (5) that the integral in time of the right hand side converges to zero and so

$$
\begin{aligned}
\lim _{j \rightarrow \infty} \int_{L_{b}^{j}} f\left(\exp \left(i \theta_{j, b}\right)\right) \phi d \mathcal{H}^{n}=\lim _{j \rightarrow \infty} \int_{L_{a}^{j}} f\left(\exp \left(i \theta_{j, a}\right)\right) \phi & d \mathcal{H}^{n} \\
& =\sum_{k=1}^{N} m_{k} f\left(\exp \left(i \bar{\theta}_{k}\right)\right) \mu_{k}(\phi)
\end{aligned}
$$

for all $b<0$.

We now prove the uniqueness statement of the theorem. Let

$$
\left(\widehat{L}_{s}^{j}\right)_{s<0}
$$

be another sequence of rescaled flows for which there are Special Lagrangian cones

$$
\widehat{L}_{1}, \ldots, \widehat{L}_{P}
$$

and a finite set $\left\{\exp \left(i \hat{\theta}_{1}\right), \ldots, \exp \left(i \hat{\theta}_{P}\right)\right\}$ such that, for every smooth function $\phi$ with compact support, every $f$ in $C^{2}\left(S^{1}\right)$, and every $s<0$,

$$
\lim _{j \rightarrow \infty} \int_{\widehat{L}_{s}^{j}} f\left(\exp \left(i \theta_{k, s}\right)\right) \phi d \mathcal{H}^{n}=\sum_{k=1}^{P} \widehat{m}_{k} f\left(\exp \left(i \hat{\theta}_{k}\right)\right) \widehat{\mu}_{k}(\phi)
$$

where $\widehat{\mu}_{k}$ and $\widehat{m}_{k}$ denote the Radon measure of the support of $L_{k}$ and its multiplicity respectively. We want to argue that

$$
\left\{\exp \left(i \hat{\theta}_{1}\right), \ldots, \exp \left(i \hat{\theta}_{P}\right)\right\}=\left\{\exp \left(i \bar{\theta}_{1}\right), \ldots, \exp \left(i \bar{\theta}_{N}\right)\right\} .
$$

For any real number $y$ and any integer $q$, we have the following evolution equation

$$
\frac{d}{d t}\left(\cos \theta_{t}-y\right)^{q}=\Delta\left(\cos \theta_{t}-y\right)^{q}+U|H|^{2},
$$

where

$$
U \equiv q\left(\cos \theta_{t}-y\right)^{q-2}\left(\left(\cos \theta_{t}-y\right) \cos \theta_{t}-(q-1) \sin ^{2} \theta_{t}\right) .
$$

Therefore

$$
\begin{array}{rl}
\frac{d}{d t} \int_{L_{t}}\left(\cos \theta_{t}-y\right)^{q} \Phi_{x_{0}, T} & d \mathcal{H}^{n} \\
& =\int_{L_{t}}\left(U|H|^{2}-\left|H-\left(\mathbf{x}-\mathbf{x}_{0}\right)^{\perp} / 2(T-t)\right|^{2} \gamma_{t}^{2}\right) \Phi_{x_{0}, T} d \mathcal{H}^{n}
\end{array}
$$

and we obtain from (3) that

$$
\lim _{t \rightarrow T} \int_{L_{t}}\left(\cos \theta_{t}-y\right)^{q} \Phi_{x_{0}, T} d \mathcal{H}^{n}
$$


exists. Hence, from scale invariance, we have for all $s, u<0$

$$
\begin{aligned}
\lim _{j \rightarrow \infty} \int_{L_{s}^{j}}\left(\cos \theta_{s}^{j}-y\right)^{q} \Phi d \mathcal{H}^{n}=\lim _{j \rightarrow \infty} \int_{\hat{L}_{u}^{j}}\left(\cos \theta_{u}^{j}\right. & -y)^{q} \Phi d \mathcal{H}^{n} \\
& =\lim _{t \rightarrow T} \int_{L_{t}}\left(\cos \theta_{t}-y\right)^{q} \Phi_{x_{0}, T} d \mathcal{H}^{n}
\end{aligned}
$$

and therefore, for all positive integer $q$ and all $y$ in $\mathbb{R}$,

$$
\sum_{k=1}^{N} m_{k}\left(\cos \bar{\theta}_{k}-y\right)^{q} \mu_{k}(\Phi)=\sum_{k=1}^{P} \widehat{m}_{k}\left(\cos \hat{\theta}_{k}-y\right)^{q} \widehat{\mu}_{k}(\Phi) .
$$

Likewise, we can use the same arguments to show that

$$
\sum_{k=1}^{N} m_{k}\left(\sin \bar{\theta}_{k}-y\right)^{q} \mu_{k}(\Phi)=\sum_{k=1}^{P} \widehat{m}_{k}\left(\sin \hat{\theta}_{k}-y\right)^{q} \widehat{\mu}_{k}(\Phi)
$$

for all positive integer $q$ and all $y$ in $\mathbb{R}$ and this implies that

$$
\left\{\exp \left(i \hat{\theta}_{1}\right), \ldots, \exp \left(i \hat{\theta}_{P}\right)\right\}=\left\{\exp \left(i \bar{\theta}_{1}\right), \ldots, \exp \left(i \bar{\theta}_{N}\right)\right\} .
$$

\section{Proof of Compactness Theorem B}

This section is devoted to the proof of Theorem B.

Theorem B. Let $L_{0}$ be a normalized monotone Lagrangian in $\mathbb{C}^{2}$ for which the Lagrangian mean curvature flow develops a singularity at time $T<1 / 2$. The following property holds for all $R>0$ and almost all $s<0$.

For any sequence $\Sigma_{j}$ of connected components of $B_{4 R}(0) \cap L_{s}^{j}$ that intersect $B_{R}(0)$, there exists a Special Lagrangian cone $\Sigma$ in $B_{2 R}(0)$ with Lagrangian angle $\bar{\theta}$ such that, after passing to a subsequence,

$$
\lim _{j \rightarrow \infty} \int_{\Sigma_{j}} f\left(\exp \left(i \theta_{j, s}\right)\right) \phi d \mathcal{H}^{n}=m f(\exp (i \bar{\theta})) \mu(\phi)
$$

for every $f$ in $C\left(S^{1}\right)$ and every smooth $\phi$ compactly supported in $B_{2 R}(0)$, where $\mu$ and $m$ denote the Radon measure of the support of $L$ and its multiplicity respectively.

Proof. The fact that $L_{0}$ is monotone implies that, for each $L_{s}^{j}$,

$$
[\lambda]=\sigma_{j}^{2}\left(1-2 T+2 s / \sigma_{j}^{2}\right)\left[d \theta_{j, s}\right] .
$$

Moreover, identity (4) implies that, after passing to a subsequence if necessary,

$$
\lim _{j \rightarrow \infty} \int_{L_{s}^{j} \cap B_{R}(0)}|H|^{2}+\left|\mathbf{x}^{\perp}\right|^{2} d \mathcal{H}^{2}=0
$$

for almost all $s<0$ and all $R>0$.

The next proposition shows that each connected components of $B_{4 R}(0) \cap L_{s}^{j}$ has a well defined Lagrangian angle and a primitive for the Liouville form. 
Proposition 5.1. For almost all $s<0$ and all $R>0$ the following property holds. Let $\left(\Sigma_{j}\right)$ be a sequence of connected components of $B_{4 R}(0) \cap L_{s}^{j}$ that intersect $B_{R}(0)$. Then, for all $j$ sufficiently large, the Lagrangian angle $\theta_{j, s}$ is well defined, there is a smooth function $\beta_{j, s}$ so that $d \beta_{j, s}=\lambda$, and

$$
\mathcal{H}^{2}\left(\Sigma_{j}\right) \geq C_{0} R^{2}
$$

where $C_{0}$ is some universal constant.

Proof. We start by showing that an isoperimetric inequality holds for almost all $s<0$.

Lemma 5.2. There is some universal constant $C$ such that, for all $j$ sufficiently large,

$$
\left(\mathcal{H}^{2}(A)\right)^{1 / 2} \leq C \mathcal{H}^{1}(\partial A)
$$

where $A$ is any open subset of $L_{s}^{j} \cap B_{6 R}(0)$ with rectifiable boundary

Proof. Recall that according to the Michael-Simon Sobolev inequality there exists a universal constant $C$ so that

$$
\left(\mathcal{H}^{2}(A)\right)^{1 / 2} \leq C \int_{A}|H| d \mathcal{H}^{2}+C \mathcal{H}^{1}(\partial A)
$$

and so

$$
\left(\mathcal{H}^{2}(A)\right)^{1 / 2} \leq C\left(\mathcal{H}^{2}(A)\right)^{1 / 2}\left(\int_{A}|H|^{2}\right)^{1 / 2}+C \mathcal{H}^{1}(\partial A) .
$$

The claim follows from (7).

Denote the intrinsic ball of radius $r$ around $x$ in $\Sigma_{j}$ by $\widehat{B}_{j}(x, r)$ and set

$$
\psi_{j}(r) \equiv \mathcal{H}^{2}\left(\widehat{B}_{j}(x, r)\right)
$$

which has, for almost all $r$, derivative given by

$$
\psi_{j}^{\prime}(r)=\mathcal{H}^{1}\left(\partial \widehat{B}_{j}(x, r)\right) .
$$

Hence, we have for all $j$ sufficiently large, all $x$ in $\Sigma_{j}$, and all $r<R$

$$
\left(\psi_{j}(r)\right)^{1 / 2} \leq C \psi_{j}^{\prime}(r)
$$

from which it follows that

$$
\mathcal{H}^{2}\left(\widehat{B}_{j}(x, r)\right) \geq C_{0} r^{2},
$$

where $C_{0}$ is some universal constant. An immediate consequence is that

$$
\mathcal{H}^{2}\left(\Sigma_{j}\right) \geq C_{0} R^{2}
$$

The lower density bounds (8) combined with the uniform upper bounds on $\mathcal{H}^{2}\left(\Sigma_{j}\right)$ imply the existence of some universal constant $k$ so that, for all $j$ sufficiently large,

$$
\operatorname{diam} \Sigma_{j} \leq k R
$$

Hence, for all $j$ sufficiently large, we have that every loop $\gamma$ in $\Sigma_{j}$ satisfies

$$
\int_{\gamma} \lambda=0
$$


because

$$
\left|\int_{\gamma} \lambda\right| \leq 4 R \text { length }(\gamma)
$$

and, due to $(6)$,

$$
\int_{\gamma} \lambda=\sigma_{j}^{2}\left(1-2 T+2 s / \sigma_{j}^{2}\right) 2 n \pi \quad \text { for some } n \in \mathbb{Z} .
$$

Note that from (6) we also obtain that

$$
\int_{\gamma} d \theta_{j, s}=0
$$

for every loop $\gamma$ in $\Sigma_{j}$ and thus the proposition is proven.

Pick $s_{0}<0$ so that both (7) and Proposition 5.1 hold, and consider a sequence $\Sigma_{j}$ of connected components of $B_{4 R}(0) \cap L_{s_{0}}^{j}$ that intersect $B_{R}(0)$. We can apply Proposition 4.1 and conclude that, after passing to a subsequence, $\Sigma_{j}$ converges weakly in $B_{2 R}(0)$ to a finite union of Special Lagrangian cones that we denote by $\Sigma$. From Proposition 5.1 we know that $\Sigma$ has positive measure. Next, using the argumentation in [4], we show that the support of $\Sigma$ is contained in the support of a Special Lagrangian cone.

According to (6), we have

$$
\left[\lambda+2\left(s-s_{0}\right) d \theta_{j, s}\right]=\left(\sigma_{j}^{2}(2 T-1)-2 s_{0}\right)\left[d \theta_{j, s}\right]
$$

and thus, we can find a sequence $\left(b_{j}\right)$ converging to one so that $b_{j}^{-1}\left(\sigma_{j}^{2}(2 T-1)-2 s_{0}\right)$ is an integer, which implies that

$$
b_{j}^{-1}\left(\lambda+2\left(s-s_{0}\right) d \theta_{j, s}\right) \in H^{1}\left(L_{s}^{j}, \mathbb{Z}\right)
$$

for all $s<0$. Hence, there is a multivalued function $\alpha_{j, s}$ so that

$$
d \alpha_{j, s}=b_{j}^{-1}\left(\lambda+2\left(s-s_{0}\right) d \theta_{j, s}\right)
$$

and

$$
u_{j, s} \equiv \cos \alpha_{j, s}
$$

is a well defined function.

Assume without loss of generality that for $s=-1$ both (7) and Proposition 5.1 hold. As a result, we have that, for all $R>0$ and all $j$ sufficiently large, both the Lagrangian angle and the primitive for the Liouville form are well defined on $L_{-1}^{j} \cap B_{2 R}(0)$.

Lemma 5.3. There is a set

$$
\left\{\left(\cos \bar{\beta}_{1}, \sin \bar{\beta}_{1}\right), \ldots,\left(\cos \bar{\beta}_{Q}, \sin \bar{\beta}_{Q}\right)\right\}
$$

of distinct pairs and integral Special Lagrangian cones

$$
P_{1}, \ldots, P_{Q}
$$


such that, after passing to a subsequence, we have for all smooth $\phi$ with compact support and all $f$ in $C(\mathbb{R})$

$$
\begin{aligned}
& \lim _{j \rightarrow \infty} \int_{L_{-1}^{j}} f\left(\cos \left(b_{j}^{-1} \beta_{j,-1}\right)\right) \phi d \mathcal{H}^{2}=\sum_{k=1}^{Q} p_{k} f\left(\cos \bar{\beta}_{k}\right) \nu_{k}(\phi) \\
& \lim _{j \rightarrow \infty} \int_{L_{-1}^{j}} f\left(\sin \left(b_{j}^{-1} \beta_{i,-1}\right)\right) \phi d \mathcal{H}^{2}=\sum_{k=1}^{Q} p_{k} f\left(\sin \bar{\beta}_{k}\right) \nu_{k}(\phi),
\end{aligned}
$$

where $\nu_{k}$ and the positive integer $p_{k}$ denote, respectively, the Radon measure of the support of $P_{k}$ and its multiplicity.

Proof. Due to Proposition 4.1, Proposition 5.1, and identity (6), the proof given in [4, Lemma 7.2] applies with no modifications.

We can combine this lemma with Proposition 4.1 and assume that, after a rearrangement of the supports of the Special Lagrangian cones and its multiplicities, for all $\phi$ with compact support, all $f$ in $C(\mathbb{R})$, and all $y \in \mathbb{R}$,

$$
\begin{aligned}
\lim _{j \rightarrow \infty} \int_{L_{-1}^{j}} f\left(\cos \left(b_{j}^{-1}\left(\beta_{j,-1}+2 y \theta_{j,-1}\right)\right)\right) \phi d \mathcal{H}^{2} & \\
& =\sum_{k=1}^{N} m_{k} f\left(\cos \left(\bar{\beta}_{k}+2 y \bar{\theta}_{k}\right)\right) \mu_{j}(\phi),
\end{aligned}
$$

where $\mu_{k}$ denotes the Radon measure of the support of $L_{k}$ and the elements of the set

$$
\left\{\left(\cos \bar{\beta}_{1}, \sin \bar{\beta}_{1}, \bar{\theta}_{1}\right), \ldots,\left(\cos \bar{\beta}_{N}, \sin \bar{\beta}_{Q}, \bar{\theta}_{N}\right)\right\}
$$

are all distinct.

According to Proposition 4.1, the functions $\alpha_{j, s}$ can be chosen so that

$$
\frac{d u_{j, s}}{d s}=\Delta u_{j, s}+u_{j, s}\left|b_{j}^{-1}\left(\mathbf{x}^{\perp}+2\left(s_{0}-s\right) H\right)\right|^{2} .
$$

This evolution equation will be used to show

Lemma 5.4. For all $\phi$ with compact support, all $f, h$ in $C^{2}(\mathbb{R})$, and all $\bar{\theta} \in \mathbb{R}$,

$$
\begin{aligned}
\lim _{j \rightarrow \infty} \int_{L_{s_{0}}^{j}} h\left(\cos \left(\theta_{j, s_{0}}-\bar{\theta}\right)\right) & f\left(\cos \left(b_{j}^{-1} \beta_{j, s_{0}}\right)\right) \phi d \mathcal{H}^{2} \\
= & \sum_{k=1}^{N} m_{k} h\left(\cos \left(\bar{\theta}_{k}-\bar{\theta}\right)\right) f\left(\cos \left(\bar{\beta}_{k}-2\left(s_{0}+1\right) \bar{\theta}_{k}\right)\right) \mu_{k}(\phi) .
\end{aligned}
$$

Proof. The proof is the same as in [4, Lemma 7.3]. Set

$$
v_{j, s} \equiv h\left(\cos \left(\theta_{j, s}-\bar{\theta}\right)\right) f\left(u_{j, s}\right) .
$$

A simple computation shows that, for $\min \left\{s_{0},-1\right\} \leq s<0$,

$$
P_{j, s} \equiv \frac{d v_{j, s}}{d s}-\Delta v_{j, s}
$$

satisfies

$$
\left|P_{j, s}\right|+\left|\nabla v_{j, s}\right|^{2} \leq C\left(H^{2}+\left|x^{\perp}\right|^{2}\right)
$$


where $C=C\left(s_{0}, f, h\right)$. Hence,

$$
\begin{aligned}
\frac{d}{d s} \int_{L_{s}^{j}} v_{j, s} \phi d \mathcal{H}^{2}=-\int_{L_{s}^{j}}\left\langle\nabla v_{j, s}, \nabla \phi\right\rangle d \mathcal{H}^{2} & +\int_{L_{s}^{j}} v_{j, s}\langle H, D \phi\rangle d \mathcal{H}^{2} \\
& +\int_{L_{s}^{j}} P_{j, s} \phi d \mathcal{H}^{2}-\int_{L_{s}^{j}} v_{j, s}|H|^{2} \phi d \mathcal{H}^{2} .
\end{aligned}
$$

Thus, it follows from (4) and (9) that the integral in time of the right hand side converges to zero and so

$$
\begin{array}{r}
\lim _{j \rightarrow \infty} \int_{L_{s_{0}}^{j}} h\left(\cos \left(\theta_{j, s_{0}}-\bar{\theta}\right)\right) f\left(\cos \left(b_{j}^{-1} \beta_{j, s_{0}}\right)\right) \phi d \mathcal{H}^{2}=\lim _{j \rightarrow \infty} \int_{L_{s_{0}}^{j}} v_{j, s_{0}} \phi d \mathcal{H}^{2} \\
=\lim _{j \rightarrow \infty} \int_{L_{-1}^{j}} v_{j,-1} \phi d \mathcal{H}^{2}=\lim _{j \rightarrow \infty} \int_{L_{-1}^{j}} h\left(\cos \left(\theta_{-1, s}-\bar{\theta}\right)\right) f\left(u_{j,-1}\right) \phi d \mathcal{H}^{2} \\
=\sum_{k=1}^{N} m_{k} h\left(\cos \left(\bar{\theta}_{k}-\bar{\theta}\right)\right) f\left(\cos \left(\bar{\beta}_{k}-2\left(s_{0}+1\right) \bar{\theta}_{k}\right)\right) \mu_{k}(\phi) .
\end{array}
$$

Without loss of generality we can assume that

$$
\cos \left(\bar{\beta}_{1}-2\left(s_{0}+1\right) \bar{\theta}_{1}\right), \ldots, \cos \left(\bar{\beta}_{N}-2\left(s_{0}+1\right) \bar{\theta}_{N}\right)
$$

are all distinct real numbers because this is true for all but countably many $s_{0}$. Moreover, we can use [4, Proposition A.1] and assume that on $\Sigma_{j}$ the sequence $\left(\cos \left(b_{j}^{-1} \beta_{j, s_{0}}\right)\right)$ converges to a constant. Let $\gamma$ be the value of this constant and define $f \in C^{2}(\mathbb{R})$ to be a nonnegative cutoff function that is one in small neighborhood of $\gamma$ and zero everywhere else.

As a result, if $\mu_{\Sigma}$ denotes the Radon measure of $\Sigma$, we can use Lemma 5.4 to conclude that, for every nonnegative test function $\phi$ with support in $B_{2 R}(0)$,

$$
\begin{aligned}
& \mu_{\Sigma}(\phi)=\lim _{j \rightarrow \infty} \int_{\Sigma_{j}} f\left(\cos \left(b_{j}^{-1} \beta_{j, s_{0}}\right)\right) \phi d \mathcal{H}^{2} \\
& \leq \lim _{j \rightarrow \infty} \int_{L_{s_{0}}^{j}} f\left(\cos \left(b_{j}^{-1} \beta_{j, s_{0}}\right)\right) \phi d \mathcal{H}^{2} \\
& \quad=\sum_{k=1}^{N} m_{k} f\left(\cos \left(\bar{\beta}_{k}-2\left(s_{0}+1\right) \bar{\theta}_{k}\right)\right) \mu_{k}(\phi) .
\end{aligned}
$$

Therefore, the fact that the support of $f$ can be made arbitrarily small and that the elements of the set

$$
\left\{\cos \left(\bar{\beta}_{1}-2\left(s_{0}+1\right) \bar{\theta}_{1}\right), \ldots, \cos \left(\bar{\beta}_{N}-2\left(s_{0}+1\right) \bar{\theta}_{N}\right)\right\} .
$$

are all distinct, implies that

$$
\gamma=\cos \left(\bar{\beta}_{k_{0}}-2\left(s_{0}+1\right) \bar{\theta}_{k_{0}}\right)
$$

for a unique $k_{0}$. As a result,

$$
\mu_{\Sigma}(\phi) \leq m_{k_{0}} \mu_{k_{0}}(\phi)
$$


for all $\phi \geq 0$, which means that the support of $\Sigma$ is contained in the support of the Special Lagrangian $L_{k_{0}}$.

In order to consider to complete the proof we need to show convergence of the Lagrangian angle. Note that this indeed requires an argument because the support of $L_{k_{0}}$ can be Special Lagrangian with two different orientations.

We know from [4, Proposition A.1] that $\left(\cos \left(b_{j}^{-1} \beta_{j, s_{0}}\right)\right)$ converges to a constant when restricted to connected components of $L_{s_{0}}^{j} \cap B_{4 R}(0)$ that intersect $B_{2 R}(0)$. Let $\tilde{\Sigma}_{j}$ denote those connected components for which $\left(\cos \left(b_{j}^{-1} \beta_{j, s_{0}}\right)\right)$ converges to $\gamma$. Using $\bar{\theta}=\bar{\theta}_{k_{0}}$ in Lemma 5.4 and keeping $f$ to be as defined above, we obtain that for every $\phi$ compactly supported in $B_{2 R}(0)$,

$$
\begin{aligned}
\lim _{j \rightarrow \infty} \int_{\tilde{\Sigma}_{j}} \cos \left(\theta_{j, s_{0}}-\bar{\theta}\right) \phi d \mathcal{H}^{2} & =\lim _{j \rightarrow \infty} \int_{L_{s_{0}}^{j}} \cos \left(\theta_{j, s_{0}}-\bar{\theta}_{k_{0}}\right) f\left(u_{j, s_{0}}\right) \phi d \mathcal{H}^{2} \\
& =\lim _{j \rightarrow \infty} \int_{L_{-1}^{j}} \cos \left(\theta_{j,-1}-\bar{\theta}_{k_{0}}\right) f\left(u_{j,-1}\right) \phi d \mathcal{H}^{2} \\
& =m_{k_{0}} \mu_{k_{0}}(\phi)=\lim _{j \rightarrow \infty} \int_{\tilde{\Sigma}_{j}} \phi d \mathcal{H}^{2}
\end{aligned}
$$

Hence, for all small $\varepsilon>0$,

$$
\lim _{j \rightarrow \infty} \mathcal{H}^{2}\left(\left\{x \in \tilde{\Sigma}_{j} \mid \cos \theta_{j, s_{0}}\left(x-\bar{\theta}_{k_{0}}\right) \leq 1-\varepsilon\right\}\right)=0
$$

and so the desired result follows.

\section{Examples}

This section is devoted to the proof of

Theorem C. There is a normalized monotone Lagrangian $L_{0}$ which is Hamiltonian isotopic to a Clifford torus and for which the Lagrangian mean curvature flow develops a singularity at the origin at time $T<1 / 2$. The rescaled flow is a transverse union of two Lagrangian planes with the same Lagrangian angle.

Proof. Given an embedded curve $\gamma$ in the complex plane, it is easy to see that

$$
L=\{(\gamma \cos \alpha, \gamma \sin \alpha) \mid \alpha \in \mathbb{R} / 2 \pi \mathbb{Z}\}
$$

is a Lagrangian in $\mathbb{C}^{2}$ which will be embedded if $\gamma$ is preserved by the antipodal map. If we evolve $L$ by mean curvature flow, then each $L_{t}$ is also rotational symmetric and the corresponding $\gamma_{t}$ evolve according to

$$
\frac{d \gamma}{d t}=\mathbf{k}-\mathbf{x}^{\perp} /|x|^{2},
$$

where $\mathbf{k}$ is the curvature of $\gamma$ and $\mathbf{x}^{\perp}$ denotes the orthogonal projection of the position vector $x$ on the orthogonal complement of $T_{x} \gamma_{t}$. A detailed study of this equation is done in [1].

In [4] we showed that the curve in $\mathbb{C}$ given by

$$
\bar{\gamma} \equiv\{s+i \mid s \in \mathbb{R}\} \cup\{s-i \mid s \in \mathbb{R}\}
$$



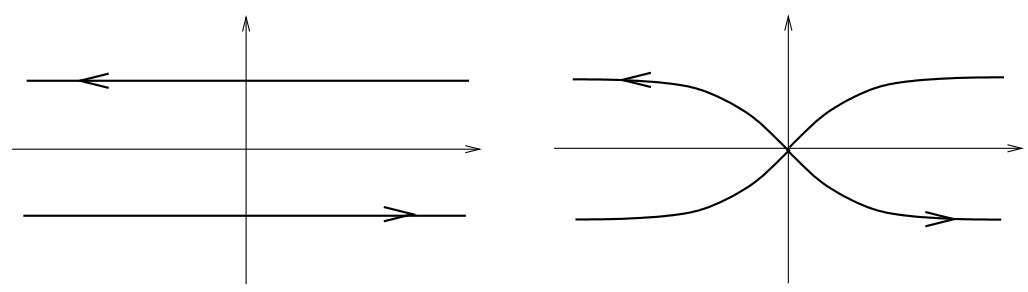

Figure $1 . \bar{L}$ at the initial time and at the time of the singularity.

(which gives rise to a zero Maslov-class Lagrangian $\bar{L}$ ) develops a finite time singularity at the origin and its tangent flow is a union of two Lagrangian planes having the same Lagrangian angle (see Figure 1).

Denote by $\gamma_{a}$ the family of ellipsoids

$$
\gamma_{a}=\left\{x+i y \in \mathbb{C} \mid x^{2} / a^{2}+y^{2} / 4 \leq 1\right\}
$$

and the correspondent family of Lagrangian torus by $L_{a}$. A simple calculation shows that, after choosing a parametrization $\gamma_{a}(s)$,

$$
\Omega_{L_{a}}=\frac{\gamma_{a}}{\left|\gamma_{a}\right|} \frac{\gamma_{a}^{\prime}}{\left|\gamma_{a}^{\prime}\right|} \operatorname{vol}_{L_{a}} \text { and } \lambda_{L_{a}}=\left\langle i \gamma_{a}, \gamma_{a}^{\prime}\right\rangle d s
$$

Hence, one can easily check that for all $a>0, L_{a}$ is monotone with the monotonicity constant $c_{a}$ equal to the area of the region enclosed by $\gamma_{a}$ divided by $2 \pi$. Denote the solution to Lagrangian mean curvature flow starting at $L_{a}$ by $\left(L_{a, t}\right)_{t \geq 0}$.

Choose $a$ large enough so that $c_{a} / 2$ is strictly bigger than $\bar{T}$, the time at which the flow $\left(\bar{L}_{t}\right)_{t \geq 0}$ with initial condition $\bar{L}$ develops a singularity. Note that for all $t \leq \bar{T}$ and while the solution exits, the region enclosed by $\gamma_{a, t}$ must contain the origin and, by Lemma 2.1, its area is greater than a positive constant. The maximum principle for smooth solutions to equation (10) implies that $\bar{\gamma}_{t}$ cannot touch $\gamma_{a, t}$ while the solution remains smooth and so $L_{a, t}$ must develop a singularity at time $T<c_{a} / 2$ (see Figure 2). The initial condition $L_{a}$ can be made normalized monotone after rescaling and in this case the singularity will occur at time $T<1 / 2$. We are left to argue that the singularity happens at the origin. This follows from either [1, Theorem 1.14] or the argument we present next.

For a short time, the curves $\gamma_{a, t}$ can be parameterized according to

$$
\gamma_{a, t}(s)=r_{t}(s) e^{i s}, \quad s \in \mathbb{R} / 2 \pi \mathbb{Z}
$$

and, as can be seen from [4, Lemma 4.6],

$$
\frac{d r}{d t}=-\frac{\theta^{\prime}}{r}=\frac{r r^{\prime \prime}-2 r^{2}-3\left(r^{\prime}\right)^{2}}{r\left(r^{\prime}\right)^{2}+r^{3}}
$$

where $\theta_{t}$ is the Lagrangian angle of $L_{a, t}$. Using the formula

$$
\theta_{0}(s)=\arg \left(\gamma_{t} \gamma_{t}^{\prime}\right)=2 s+\arg \left(r_{0}^{\prime}+i r_{0}\right),
$$

one can check that $\theta_{0}^{\prime}(s) \geq 0$. Thus, arguing in the same way as in [4, Lemma 5.2], we obtain 

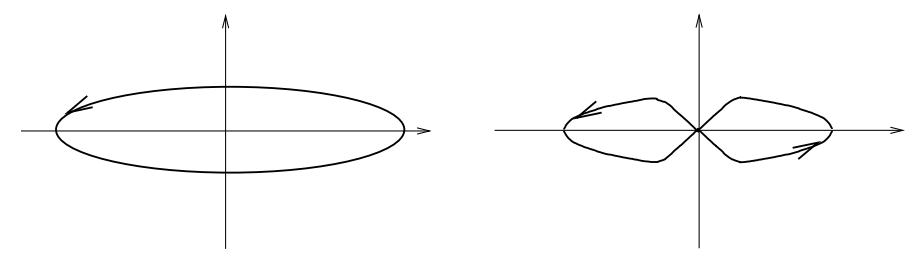

Figure 2. $L_{a}$ at the initial time and at the time of the singularity.

Lemma 6.1. While the solution to (11) exists smoothly,

$$
\frac{d r}{d t} \leq 0
$$

If a singularity happens for equation (11) at time $t_{1}$, then Lemma 6.1 implies that the curve $\gamma_{t_{1}, a}$ can be parameterized as $r_{t_{1}}(s) e^{i s}$. Thus, $t_{1}$ must also equal $T$ (the singularity time (10)) because otherwise $\gamma_{t_{1}, a}$ would be smooth and so $r_{t_{1}}(s)$ would also be smooth.

Lemma 6.2. For any $t<T, r_{t}(s)$ is nonincreasing when either $0 \leq s \leq \pi / 2$ or $\pi \leq s \leq 3 \pi / 2$, and nondecreasing when either $\pi / 2 \leq s \leq \pi$ or $3 \pi / 2 \leq s \leq 2 \pi$.

Proof. Direct computation shows that, denoting $r_{t}^{\prime}$ by $u_{t}$,

$$
\frac{d u_{t}}{d t}=\frac{u_{t}^{\prime \prime}}{\left(r^{\prime}\right)^{2}+r^{2}}+u_{t}^{\prime} b\left(r_{t}, u_{t}, u_{t}^{\prime}\right)+u_{t} c\left(r_{t}, u_{t}, u_{t}^{\prime}\right),
$$

where the functions $b$ and $c$ are bounded for each $t<T$.

Because $\gamma_{t, a}$ is symmetric with respect to the coordinate axes, it is immediate to recognize that

$$
u_{t}(0)=u_{t}(\pi / 2)=u_{t}(\pi)=u_{t}(3 \pi / 2)=0
$$

for all $t<T$. The result follows from the maximum principle.

Suppose now that the singularity for the flow happens at a point $x_{0}=r e^{i \alpha}$ with $r>0$. From Theorem A we know that the tangent flow at the singularity is a union of planes and so, by White's regularity Theorem [7],

$$
\limsup _{\delta \rightarrow 0} \frac{\mathcal{H}^{1}\left(\gamma_{T-\delta^{2}} \cap B_{\delta}\left(x_{0}\right)\right)}{2 \delta} \geq 2 .
$$


This is impossible because, using Lemmas 6.1 and 6.2, we can argue like in [4, Theorem 4.1] and conclude that, for all $\delta$ sufficiently small and all $t<T$,

$$
\frac{\mathcal{H}^{1}\left(\gamma_{t} \cap B_{\delta}\left(x_{0}\right)\right)}{2 \delta} \leq 3 / 2
$$

\section{References}

[1] K. Groh, M. Schwarz, K. Smoczyk, and K. Zehmisch, Mean curvature flow of monotone Lagrangian submanifolds, (2006), preprint.

[2] R. Harvey and H. B. Lawson, H. Calibrated geometries, Acta Math. 148 (1982), 47-157.

[3] G. Huisken, Asymptotic behavior for singularities of the mean curvature flow, J. Differential Geom. 31 (1990), 285-299.

[4] A. Neves, Singularities of Lagrangian mean curvature flow: Zero-Maslov-class case, to appear in Inventiones.

[5] R. Schoen and J. Wolfson, Minimizing area among Lagrangian surfaces: the mapping problem, J. Differential Geom. 58 (2001), 1-86.

[6] K. Smoczyk, Angle theorems for the Lagrangian mean curvature flow, Math. Z. 240 (2002), 849-883.

[7] B. White, A local regularity theorem for mean curvature flow, Ann. of Math. 161 (2005), $1487-1519$.

Fine Hall, Princeton University, Princeton, NJ 08544, USA

E-mail address: aneves@math.princeton.edu 